\title{
Android-based Voice Activated Dot Matrix Display
}

\author{
Sathis Vatumalai ${ }^{1}$ and A. Oudah ${ }^{2}$ \\ Faculty of Manufacturing Engineering, Universiti Malaysia Pahang \\ sathis.vatu@gmail.com ${ }^{1}$, multicore.processor @yahool.com ${ }^{2}$
}

\begin{abstract}
The most common problem associated with LED displays arises from reprogramming it. Most of off-the-shelf ones are somewhat inflexible in terms of updating the message instantly as well as having to re-update messages periodically through wired connection where the user is required to use a computer to change the displayed messages. In this article, an Android operating system controlled wireless dictated dot matrix display is designed and implemented. The proposed display eliminates the need to have a wired connection and requires only a smart phone to reprogram it.
\end{abstract}

Keywords: Dot matrix display, speech to text, wireless

\section{Introduction}

In this modern world full with vast growing technology, information is being transfer and convey into many form. Each of the information is conveyed by its specific need, like a computer server that store massive data that can be excess throughout the world in [1-2]. Voice or speech recognition is the ability of a machine or program to receive and interpret dictation, or to understand and carry out spoken commands. As for this device, the information is being visually transmitted from the dot matrix display straight to the viewers by using voice recognition.

The necessary design and the creation of the remote programming device for LED display using an android OS system interface which includes the following component: Smart phone, LED display, Bluetooth module, Arduino microcontroller and a basic circuit [3-4]. The project is aimed to be used as an alternative way to program the LED display to ease the user the trouble to bring the display panel down from its usually high positioned place to the point that the LED display can be program from afar as the Bluetooth range can support.

The most common problem using the LED display comes when to reprogramming it [5]. But these off-the-shelf units are somewhat inflexible in terms of updating the message instantly. If the user wants to change the message it needs to be done using a computer and the person needs to be present at the location of the display board. Furthermore, all voice-recognition systems or programs make errors. Screaming children, barking dogs, and loud external conversations can produce false input. There is also a problem with words that sound alike but are spelled differently and have different meanings.

The aim for this work is to design an LED display that can be used and programmed by any user of android smart phone throughout an application that is specifically developed to use the Bluetooth capability of the phone and communicate with another Bluetooth module thus remotely program the LED display using a voice to send the messages. 


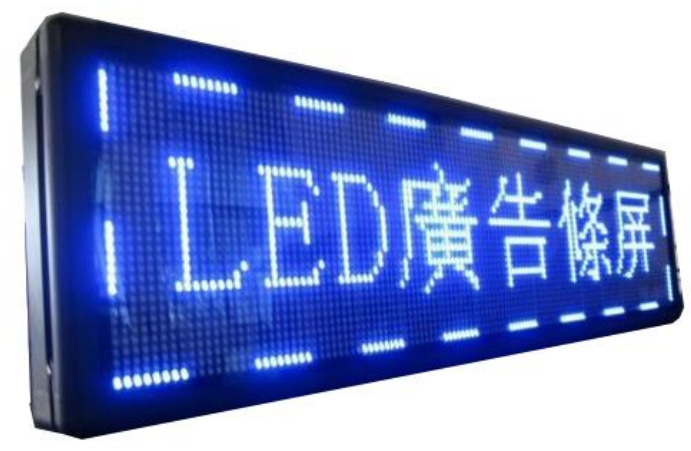

Figure 1. Commercial Dot Matrix Display [1]

\section{Proposed System}

\section{a) Type Of Microcontroller}

There are many type of micro-controller available in this vast technology spreading with a lot of function. Two of the micro-controller giants are the PIC and AVR. Both companies produce a similar product with their own set of IDE compiler. Atmel AVR controllers are lot faster and superior to PIC controllers. AVR have much better development tools (compilers, IDE, debugging tools, etc.,).AVR in had release a highly adaptive single board micro-controller that called Arduino. In this research, the chosen micro-controller is Arduino series as it is simple to understand.

b) Driver Circuit

A multiplexer is needed in order to drive all 64 LED light in a single dot-matrix and to reduce the amount of pin needed to control the LEDs. It would take a 64 individual pin from Arduino to control a single panel without a led driver. The project is to make a scrolling text, using voice recognition, the uses at least 7 panel of led used in the project totaling up to 448 LEDs that need to be control at the same time. An LED driver is important and the most suitable component to be used to handle this condition. A Max7219 chip can be used to control all 64 LED at the same time while just using a single pin out from the Arduino.

The max 7219 can set the 64 LEDs independently and by cycling through the row it will look like all the led all lit at the same time. The Max7219 chips have constant-current drivers there for eliminating the need of separate current limiting resistor on every LED. The current drive is set using one resistor as the value is different and in this case the resistor connected to the ISET pin is connected to Vdd (power). The 2.1 Table show the current-setting resistor values for the Max7219 chips shown in Figure 2.1.

Table 1. RSET vs. Segment Current and LED Forward Voltage

\begin{tabular}{|c|c|c|c|c|c|}
\hline \multirow{2}{*}{ ISEG (mA) } & \multicolumn{5}{|c|}{ VLED (V) } \\
\cline { 2 - 6 } & $\mathbf{1 . 5}$ & $\mathbf{2 . 0}$ & $\mathbf{2 . 5}$ & $\mathbf{3 . 0}$ & $\mathbf{3 . 5}$ \\
\hline 40 & 12.2 & 11.8 & 11.0 & 10.6 & 9.69 \\
\hline 30 & 17.8 & 17.1 & 15.8 & 15.0 & 14.0 \\
\hline 20 & 29.8 & 28.0 & 25.9 & 24.5 & 22.6 \\
\hline 10 & 66.7 & 63.7 & 59.3 & 55.4 & 51.2 \\
\hline
\end{tabular}




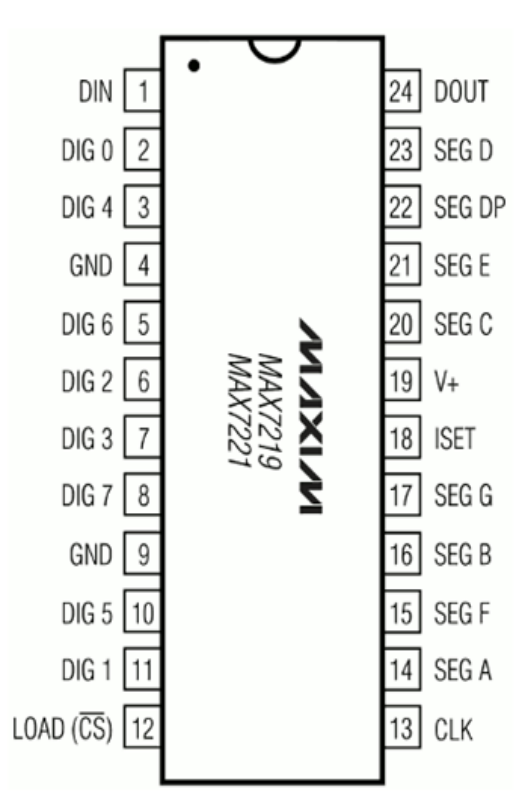

\section{Figure 2. Max7219 Pin Out}

c) Speech to Text Conversion using Android Platform

Speech not usually been used much in the field of electronics and computers due to the difficulty and diversity of the speech signals and sounds. However, with modern processes, algorithms, and methods the process of speech signals can easily and recognize the text. To make a development of an on-line speech-to-text engine. The system need speech at run time through a microphone and processes the sampled speech to recognize the uttered text. . The developing of this on android the platform using eclipse workbench. The speech-to-text system directly acquires and change the speech to text. A speech-to-text system can also be access by providing data entry options for physically handicapped users. Voice note is an application developed in this work that allows a user to record and convert spoken messages into SMS text message. . Speech recognition is done via the Internet, connecting to Google's server. The application is adjust to input messages in English. The technique used based on hidden Markov models (HMM - Hidden Markov Model) for Speech recognition for Voice. It is currently the most successful and most flexible approach to speech recognition.

\section{Methodology}

This section describes the whole project development process along with its requirements. The methods of how the project being designed and fabricated from the electrical view up until its programming method. The detail process of the project will be shown on the flow chart in Figure 1, below. 


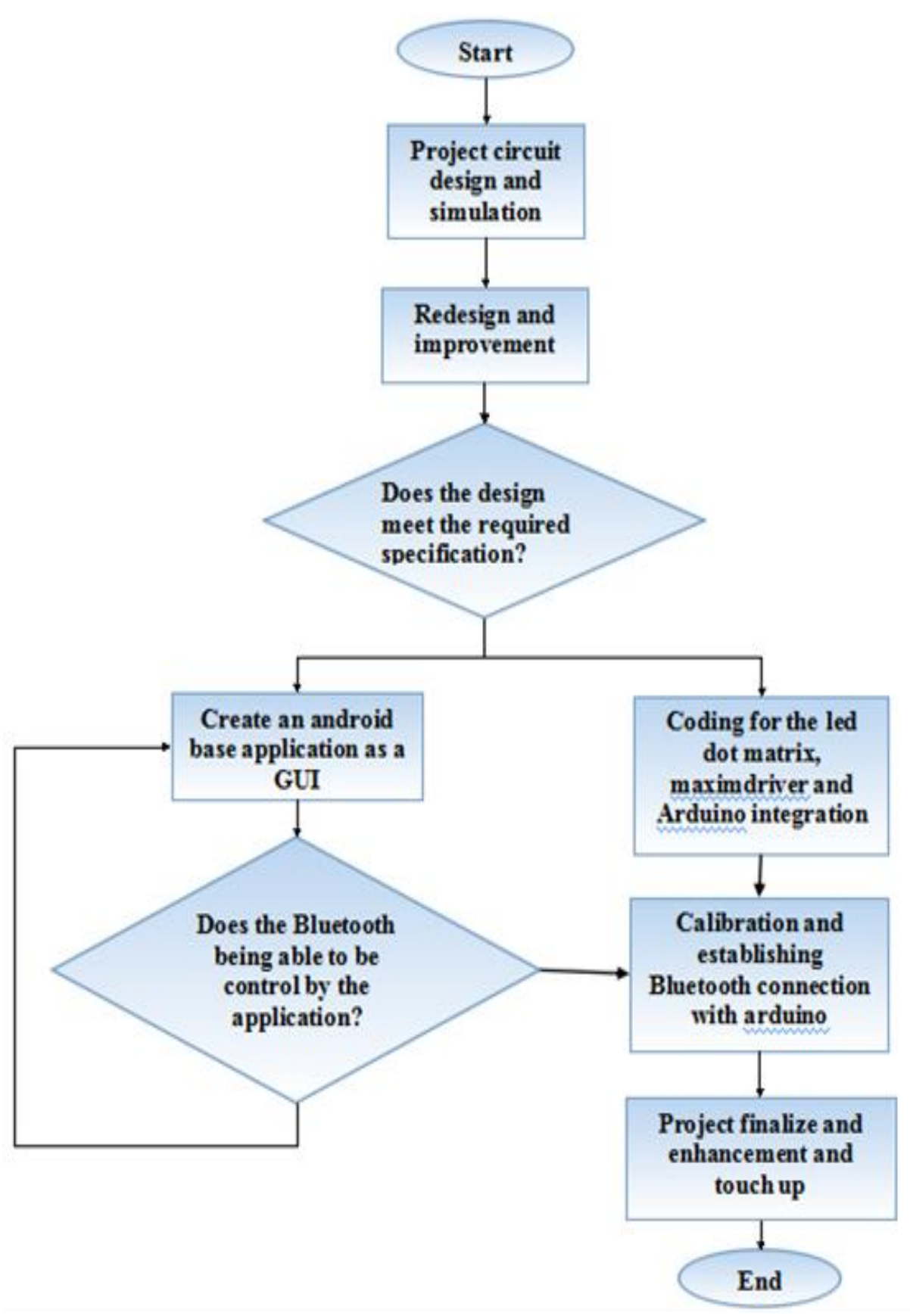

Figure 3. Flowchart of the Project

a) Electrical Design

In this section, a detailed descriptions of the component used in the project are discuss and describe. This is to show it's significant to the project and on why the component is chosen to be use. 


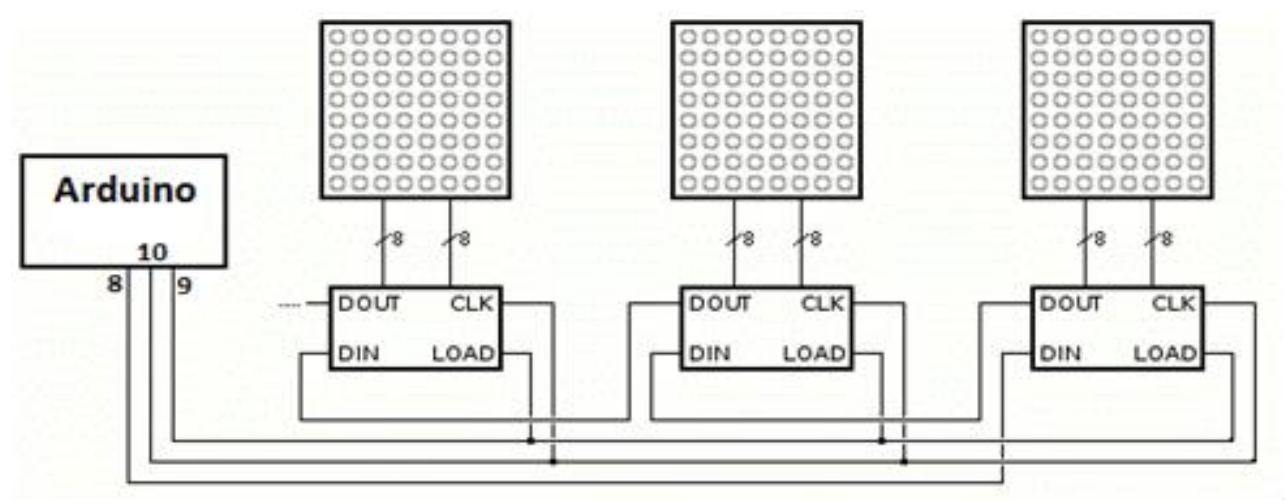

Figure 4. Basic Design of the Driver Circuit in Daisy Chain

A daisy chain is an interconnection of computer devices, peripherals or network nodes in series of one after another. In this project the dot-matrix were connected in series in daisy chain.

\section{b) MAX7219 LED Driver}

The Maxim MAX7219 driver is a a driver that capable of driving a maximum 64 LED individually or a total of 8 7-segment numeric LED display plus a multiple bar graph LED. The most crucial feature of the driver is that it can be use through SPI (Serial Peripheral Interface) that make it possible to be used with Arduino Uno. The driver can give the ability to analogue and digitally control the brightness of the LED thus makes it very suitable to be used in the project.

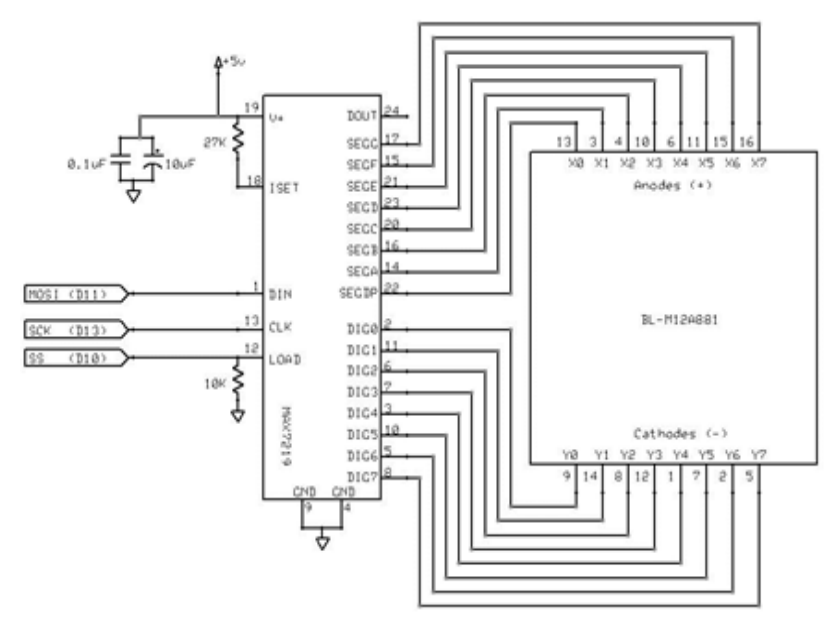

Figure 5. MAX7219 Connections to the $8 \times 8$ Dot- Matrix

The MAX7219 operate by using a Data In pin that uses 16-bit packet to determine which LED to be light on and off. The data then can be shifted to the next driver that execute the same command but with a delay. The delay is the amount of time taken by the previous driver to execute the command given then the next driver would continuously perform the same command right after the 1st driver as the shift register in the driver shift the data to create the scrolling word effect.

\section{Computing Power Dissipation}

The upper limit for power dissipation (PD) for the the project MAX7219/MAX7221 is determined from the following equation: 
$\mathrm{PD}=(\mathrm{V}+\mathrm{x} 8 \mathrm{~mA})+(\mathrm{V}+-\mathrm{VLED})($ DUTY $\mathrm{x}$ ISEG $\mathrm{x} \mathrm{N})$

where:

$\mathrm{V}+=$ supply voltage

DUTY $=$ duty cycle set by intensity register

$\mathrm{N}=$ number of segments driven (worst case is 8)

VLED = LED forward voltage

ISEG $=$ segment current set by RSET

Dissipation for the project:

ISEG $=40 \mathrm{~mA}$,

$\mathrm{N}=8$,

DUTY $=31 / 32$,

$\mathrm{VLED}=1.8 \mathrm{~V}$ at

$40 \mathrm{~mA}, \mathrm{~V}+=5.25 \mathrm{~V}$

$\mathrm{PD}=(5.25 \mathrm{~V} \times 8 \mathrm{~mA})+(5.25 \mathrm{~V}-1.8 \mathrm{~V})(31 / 32 \times$

$40 \mathrm{~mA} \times 7)=0.98 \mathrm{~W}$

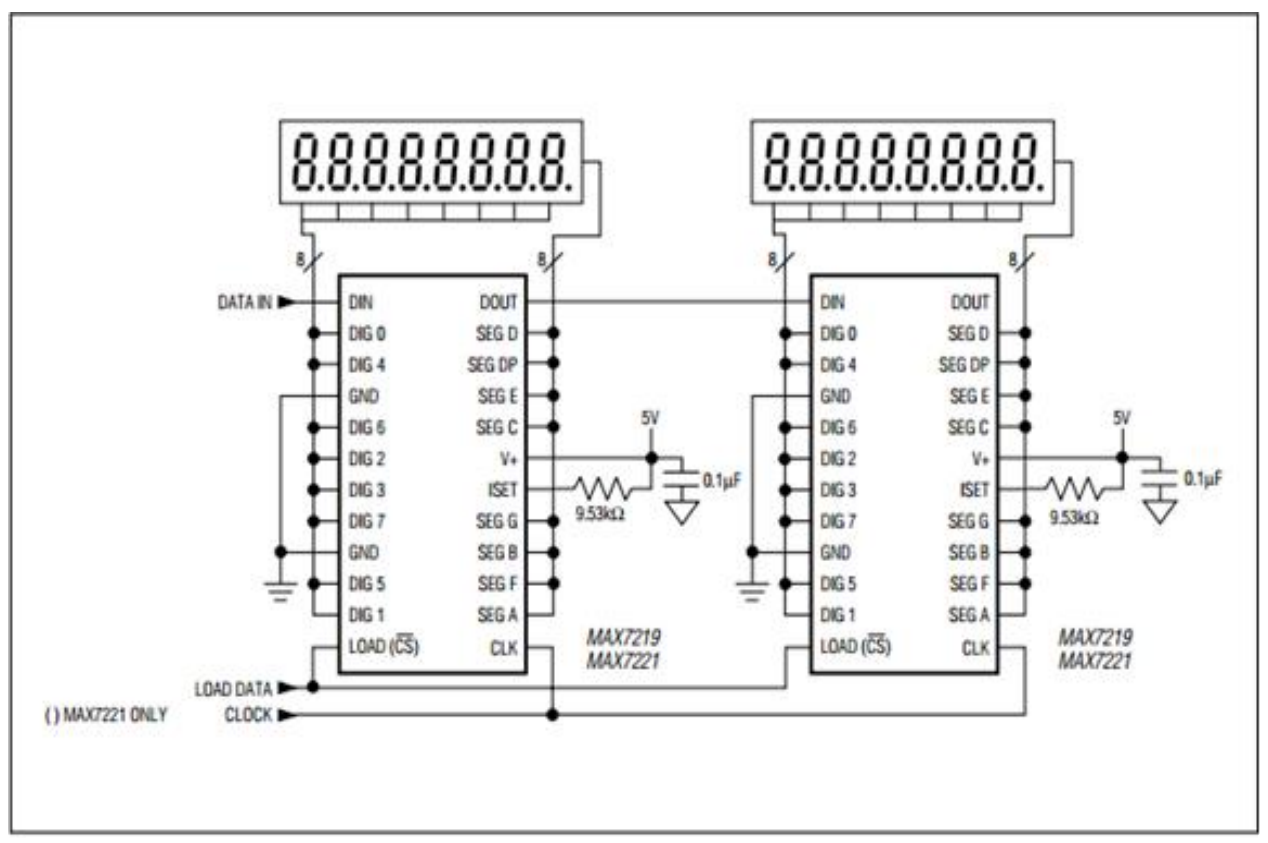

Figure 6. Cascading MAX7219/MAX7221s to Drive 16 Seven-Segment LED Digits

Cascading the drivers for the Max7219. If the number of digits is not a multiple of 8 , set both drivers' scan limits registers to the same number so one display will not appear brighter than the other. For example, if 12 digits are need, use 6 digits per display with both scan-limit registers set for 6 digits so that both displays have a 1/6 duty cycle per digit. If 11 digits are needed, set both scan-limit registers for 6 digits and leave one digit driver unconnected. If one display for 6 digits and the other for 5 digits, the second display will appear brighter because its duty cycle per digit will be $1 / 5$ while the first display's will be $1 / 6$.

\section{c) $8 \times 8$ Dot- Matrix Display}

The 8x8 LED dot matrix is use as a scrolling sign projecting words in order to have a suitable output for the signal being transmit via Bluetooth between the Bluetooth module and the android phone as its moving from side to side to convey the message sent by the phone. A panel of the 8x8 LED dot matrix consists of 64 LED with the pin arrangement of 8 pin on the top and bottom of the dot matrix. The 
LED will be individually controlled by the LED driver that does the work of multiplexing on its own.

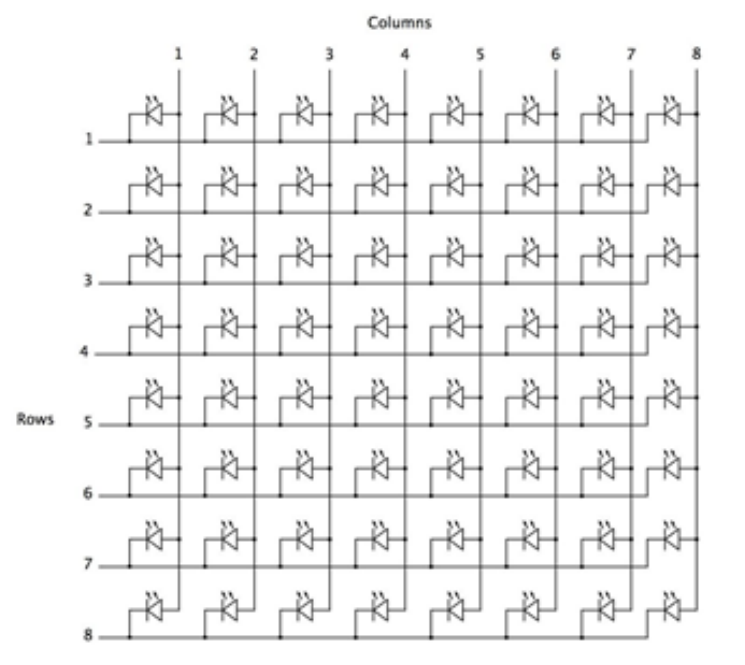

Figure 7. 8x8 Dot Matrix Internal Circuit

d) Bluetooth Module (HC-06)

The crucial part of the project is the establishment of the Bluetooth communication between the Arduino and an android phone. The Bluetooth module use is a small form module that utilize a low power Bluetooth radio module with a connect pin of $2 \times 10$ socket. The module is powered by $3.3 \mathrm{~V}$ with a small $30 \mathrm{~mA}$ to transmit. The reason to use this type of module is due to the UART data connection interface that is a capability known to be used in Arduino. The module is a class 2 Bluetooth module that capable to be use in range up to 20 meters.

HC-05 module is an easy to use Bluetooth SPP (Serial Port Protocol) module, designed for transparent wireless serial connection setup.

Serial port Bluetooth module is fully qualified Bluetooth V2.0+EDR (Enhanced Data Rate) $3 \mathrm{Mbps}$ Modulation with complete $2.4 \mathrm{GHz}$ radio transceiver and baseband. It uses CSR Bluecore 04-External single chip Bluetooth system with CMOS technology and with AFH(Adaptive Frequency Hopping Feature). It has the footprint as small as $12.7 \mathrm{mmx} 27 \mathrm{~mm}$.

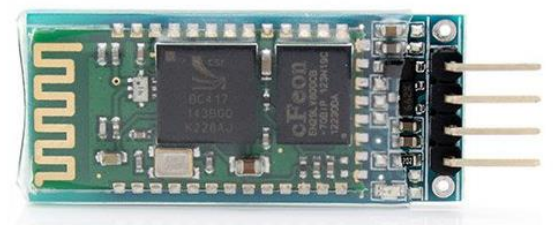

Figure 8. Bluetooth Module HC-06

\section{e) Arduino UNO R3}

The brain behind all the component combine and being controlled using an Arduino Uno. It is a microcontroller that has the capability to give the signal in terms of data to the MAX7219 driver and process through its RX and TX pin out to communicate with the android phone through the Bluetooth module. It's operating voltage of $5 \mathrm{~V}$ and a special pin out that supply a $3.3 \mathrm{~V}$ that is needed for the 
Bluetooth module makes the Arduino the best choice to run the project with, along with simplifying the overall project and circuit.

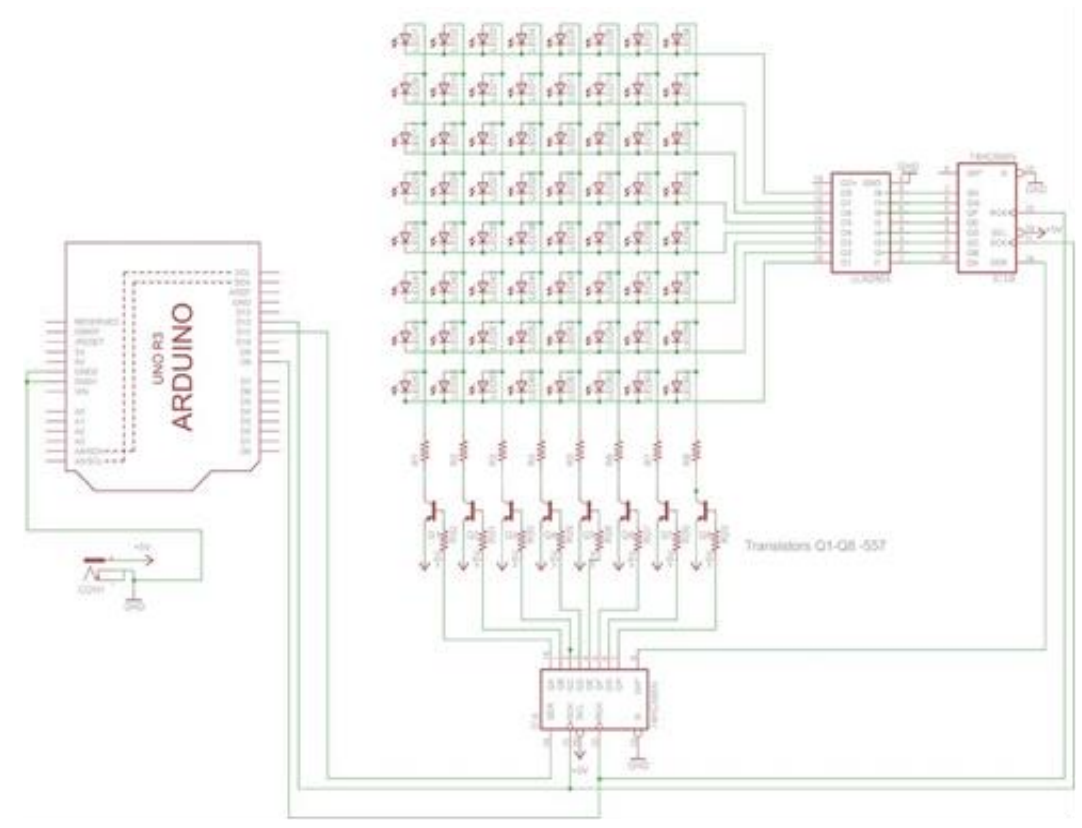

\section{Figure 9. An Example of Arduino Running an 8x8 Dot Matrix Display Schematic}

The Arduino also simplify the need for advance and complicated coding to run both MAX7219 driver and the Bluetooth module. The connection between the Arduino Bluetooth module and the android phone must first be establish and pair with. After the pairing complete then will the data key-in by the user can be sent via Bluetooth and being interpreted by the Arduino and projected through the $8 \times 8$ LED dot matrix.

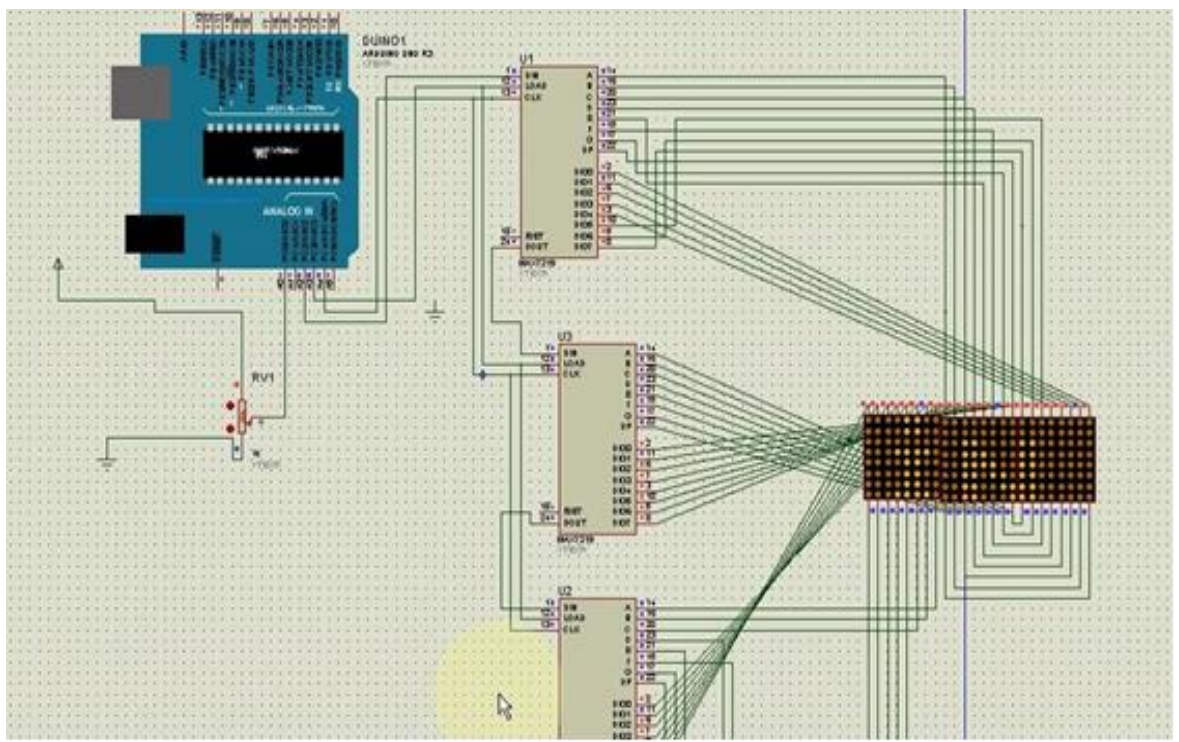

Figure 10. MAX7219 Being Run by Arduino Simulation

f) Software Integration 
Multiple software are needed to be used to be able to run the project and achieve the desired outcome. In this project the software that being use are Arduino IDE and also Proteus for the simulation of the circuit to test either the led dot-matrix with its driver.as the app software are the android meet robot app which being used as a medium of the Bluetooth module and smart phone Bluetooth to convey the data. The Figure 11, shows the flow of the programming method in the project.

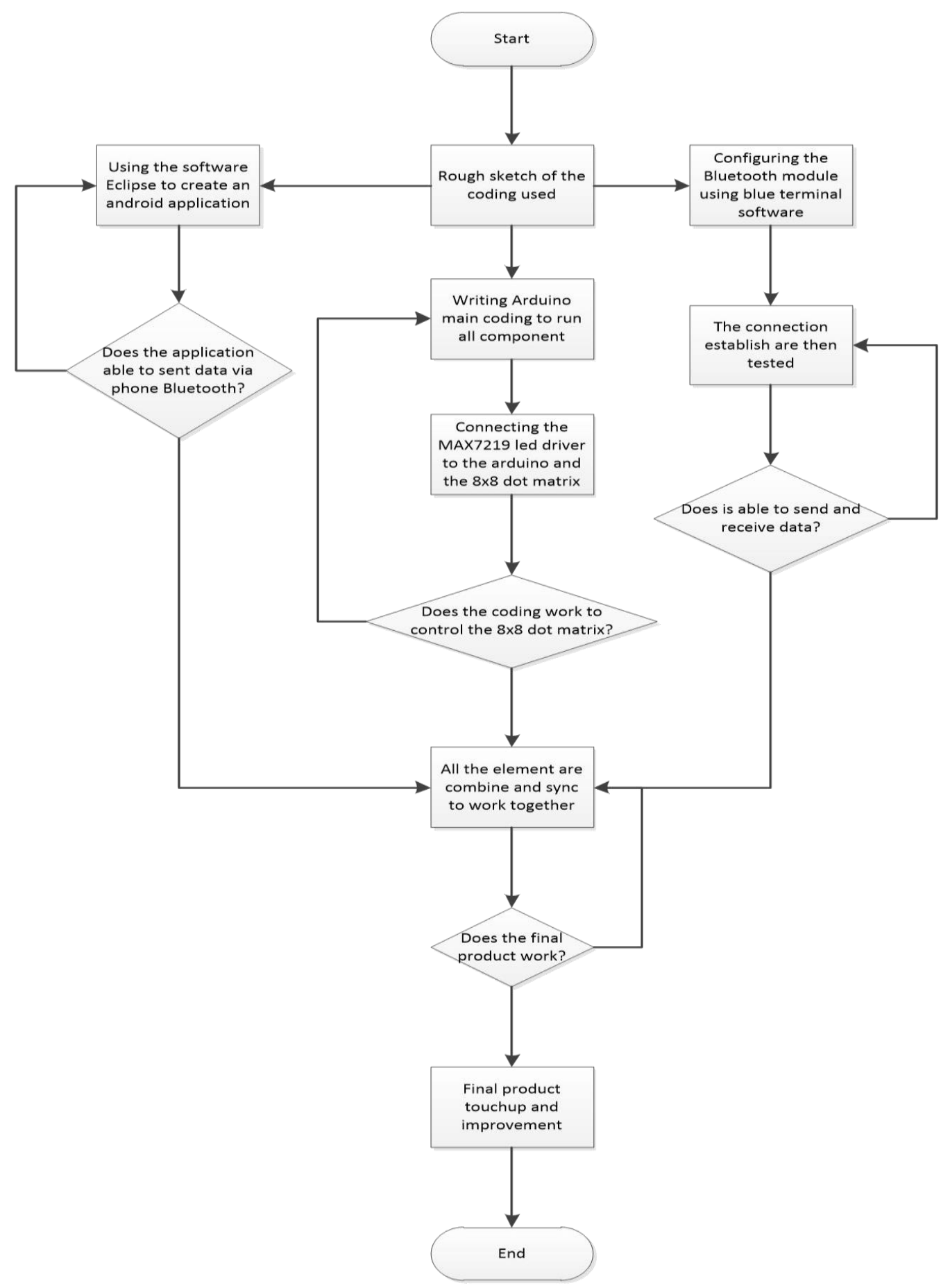

Figure 11. Detailed Programming Outline Flowchart 


\section{Results and Discussion}

With the completion of this project, Figures $12, \& 13$, the display is able to properly display the intended voice recognition and automatically scrolling them through the LED-dot matrix display. The LED dot matrix display is very versatile display and a robust device that had been around through this vast spreading technology world, and yet the way it has been use does not change a lot. Usually the display is being program through wired data transfer. Many of them produce the display with different kind of software to run them, while not all of them are working with one another. The usage of this project would standardize the way it being reprogrammed.
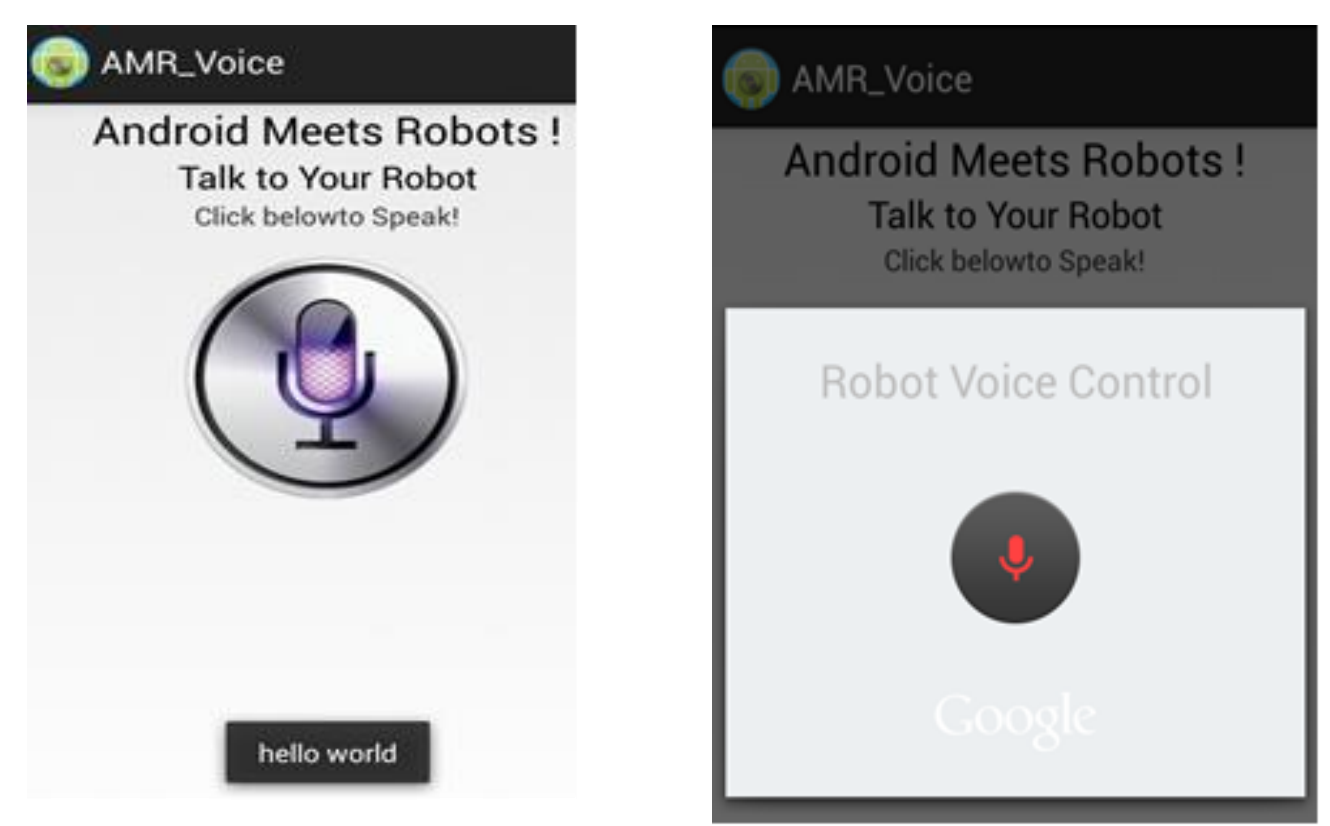

Figure 12. The Designed Android App Where it Shows the Text before Sending it to the Display

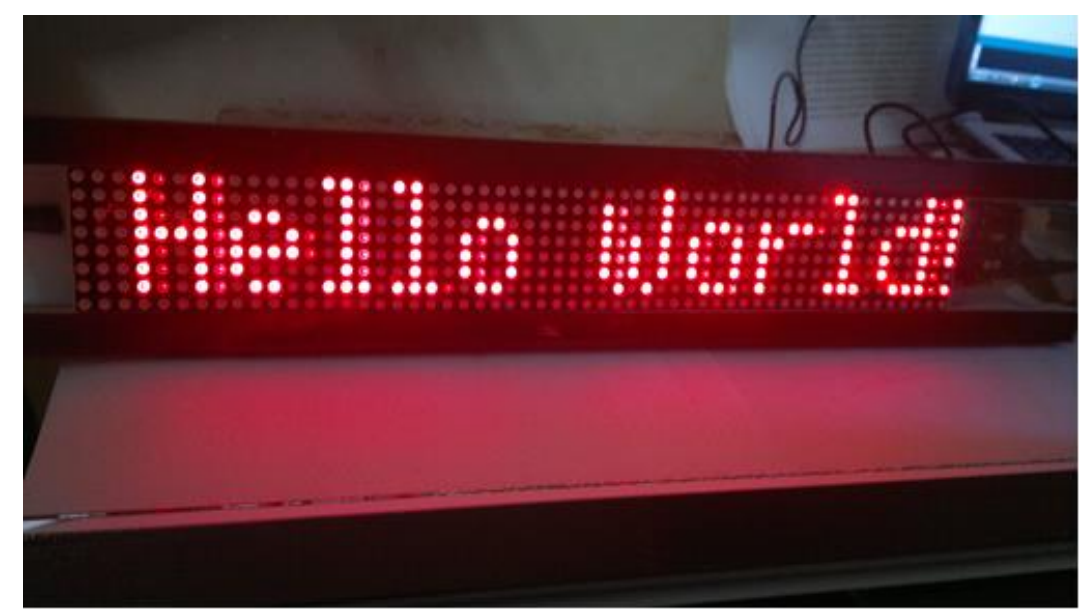

Figure 13. LED Dot-Matrix Display Showing the Intended Spoken Words 
This project would eliminate the need to even physically touch the display that is probably placed on a higher place. The app was able to reprogram the display from afar through its Bluetooth connection.

The objective is to integrate an android application that able to access the Bluetooth capabilities in the phone was a success the app is able to turn on the Bluetooth present on the phone and initiate the connection with other Bluetooth. The app had successfully control the Arduino through the RX/TX modulation thus being able to reprogram the display.

\section{Conclusions}

A new wireless dot matrix display has been designed and fabricated in this work. Serial data from an android phone to the dot display through Bluetooth link was sent successfully. The human voice was converted to text and wirelessly sent to the dot display without the need to physically touch the display.

\section{References}

[1] J. Akita, "Matrix LED unit with pattern drawing and extensive connection", In ACM SIGGRAPH 2010 Emerging Technologies on - SIGGRAPH '10 http://doi.org/10.1145/1836821.1836838, pp. 1-1, (2010).

[2] www.ijera.com/papers/Vol3_issue1/AJ31253258.pdf.http://www.ijera.com/papers/Vol3_issue1/AJ31253 258.pdf .

[3] Easy bluetooth controlled scrolling text - All.http://www.instructables.com/id/Easy-bluetoothcontrolled-scrolling-text/?ALLSTEPS.

[4] Adafruit customer service forums - View topic - 8x8 LED Matrix. Length of for-loop when scrolling text?.http://forums.adafruit.com/viewtopic.php?f=47\&p=200983

[5] K. Baraka, M. Ghobril, S. Malek, R. Kanj and A. Kayssi, "Low cost arduino/android-based energyefficient home automation system with smart task scheduling", Proceedings-5th International Conference on Computational Intelligence, Communication Systems, and Networks, CICSyN 2013, 296-301. http://doi.org/10.1109/CICSYN.2013.47, (2013). 
International Journal of Grid and Distributed Computing

Vol. 9, No. 10 (2016) 